OPEN ACCESS

Edited by:

Elisa Borghi,

University of Milan, Italy

Reviewed by:

Zhendong Cai,

Ningbo University, China Edoardo Pasolli,

University of Trento, Italy

Claudio Tiribelli,

Italian Liver Foundation ONLUS, Italy

*Correspondence:

Karl-Heinz Wagner

karl-heinz.wagner@univie.ac.at

Specialty section:

This article was submitted to Microbiome in Health and Disease,

a section of the journal

Frontiers in Cellular and

Infection Microbiology

Received: 28 April 2021

Accepted: 30 August 2021

Published: 16 September 2021

Citation:

Zöhrer PA, Hana CA Seyed Khoei N, Mölzer C, Hörmann-Wallner M, Tosevska A, Doberer D, Marculescu R,

Bulmer AC, Herbold CW, Berry $D$ and Wagner K-H (2021)

Gillbert's Syndrome and the

Gut Microbiota - Insights

From the Case-Contro

BILIHEALTH Study.

Front. Cell. Infect. Microbiol. 11:701109.

doi: 10.3389/fcimb.2021.701109

\section{Gilbert's Syndrome and the Gut Microbiota - Insights From the Case-Control BILIHEALTH Study}

\author{
Patrick A. Zöhrer ${ }^{1,2}$, Claudia A. Hana ${ }^{1}$, Nazlisadat Seyed Khoei ${ }^{1,2}$, Christine Mölzer ${ }^{3}$, \\ Marlies Hörmann-Wallner ${ }^{4}$, Anela Tosevska ${ }^{1,2,5}$, Daniel Doberer ${ }^{6}$, Rodrig Marculescu ${ }^{7}$, \\ Andrew C. Bulmer ${ }^{8}$, Craig W. Herbold ${ }^{9}$, David Berry ${ }^{9,10}$ and Karl-Heinz Wagner ${ }^{1,2^{*}}$ \\ ${ }^{1}$ Department of Nutritional Sciences, Faculty of Life Sciences, University of Vienna, Vienna, Austria, ${ }^{2}$ Research Platform \\ Active Ageing, University of Vienna, Vienna, Austria, ${ }^{3}$ School of Medicine, Medical Sciences and Nutrition, Institute of Medical \\ Sciences, University of Aberdeen, Aberdeen, United Kingdom, ${ }^{4}$ Institute for Dietetics and Nutrition, University of Applied \\ Sciences FH JOANNEUM, Graz, Austria, ${ }^{5}$ Division of Rheumatology, Department of Medicine 3, Medical University of \\ Vienna, Vienna, Austria, 6 Clinical Institute of Laboratory Medicine, Vienna General Hospital, Medical University of Vienna, \\ Vienna, Austria, ${ }^{7}$ Department of Clinical Pharmacology, Vienna General Hospital, Medical University of Vienna, Vienna, Austria, \\ ${ }^{8}$ School of Pharmacy and Medical Sciences and Menzies Health Institute Queensland, Griffith University, Gold Coast, QLD, \\ Australia, ${ }^{9}$ Division of Microbial Ecology, Department of Microbiology and Ecosystem Science, Centre for Microbiology and \\ Environmental Systems Science, University of Vienna, Vienna, Austria, ${ }^{10}$ Joint Microbiome Facility of the Medical University of \\ Vienna and University of Vienna, Vienna, Austria
}

The heme catabolite bilirubin has anti-inflammatory, anti-oxidative and anti-mutagenic effects and its relation to colorectal cancer (CRC) risk is currently under evaluation. Although the main metabolic steps of bilirubin metabolism, including the formation of stercobilin and urobilin, take place in the human gastrointestinal tract, potential interactions with the human gut microbiota are unexplored. This study investigated, whether gut microbiota composition is altered in Gillbert's Syndrome (GS), a mild form of chronically elevated serum unconjugated bilirubin (UCB) compared to matched controls. Potential differences in the incidence of CRC-associated bacterial species in GS were also assessed. To this end, a secondary investigation of the BILIHEALTH study was performed, assessing 45 adults with elevated UCB levels (GS) against 45 age- and sex-matched controls (C). Fecal microbiota analysis was performed using $16 \mathrm{~S}$ rRNA gene sequencing. No association between mildly increased UCB and the composition of the gut microbiota in this healthy cohort was found. The alpha and beta diversity did not differ between $\mathrm{C}$ and GS and both groups showed a typical representation of the known dominant phyla. Furthermore, no difference in abundance of Firmicutes and Proteobacteria, which have been associated with the mucosa of CRC patients were observed between the groups. A sequence related to the Christensenella minuta strain YIT 12065 was identified with a weak association value of 0.521 as an indicator species in the GS group. This strain has been previously associated with a lower body mass index, which is typical for the GS phenotype. Overall, sex was the only driver for an identifiable difference in the study groups, as demonstrated by a greater bacterial diversity in women. 
After adjusting for confounding factors and multiple testing, we can conclude that the GS phenotype does not affect the composition of the human gut microbiota in this generally healthy study group.

Keywords: bilirubin, UGT1A1, unconjugated bilirubin, 16S rRNA gene, microbiota, microbiome, colorectal cancer

\section{INTRODUCTION}

Mild hyperbilirubinemia, a benign condition also known as Gilbert's Syndrome (GS), is usually defined by an unconjugated bilirubin (UCB) blood concentration of above $17.1 \mu \mathrm{mol} / \mathrm{L}$. The prevalence of GS is remarkably common, affecting $5-10 \%$ (depending on ethnicity and sex) of the adult population (Wagner et al., 2018). This condition is influenced by a combination of increased haem catabolism and various underlying promoter polymorphisms in the uridine diphosphoglucuronyltransferase (UGT1A1) gene, leading to reduced conjugating activity of this enzyme and therefore, elevated UCB levels. GS is currently assumed to have little or no pathological consequences (Bulmer et al., 2018). A compelling body of evidence has demonstrated that serum bilirubin, a byproduct of hemoglobin breakdown, has substantial anti-inflammatory, anti-oxidative and antimutagenic properties (Stocker, 2004; Bulmer et al., 2008; Vitek and Tiribelli, 2020) and that mildly elevated serum bilirubin levels are strongly associated with a reduced prevalence of chronic diseases, such as CVD, Type-2 diabetes and some cancers (Zucker et al., 2004; Wagner et al., 2015; Bulmer et al., 2018; Kwon et al., 2018).

One common link between reduced disease risk and increased UCB concentration is reduced body weight, with consistent reports in the literature demonstrating significantly reduced BMI and occasionally also reduced fat mass in GS when compared to age- and sex-matched controls (Bulmer et al., 2013; Wallner et al., 2013c; Seyed Khoei et al., 2018).

UCB is formed from the breakdown of haem-containing proteins (principally hemoglobin) in the liver/spleen by heme oxygenase, resulting in biliverdin and further enzymatic transformation by biliverdin reductase to bilirubin. Unconjugated bilirubin is removed from the blood by the liver and conjugated by UGT1A1. Conjugated bilirubin is then transported to the bowel via the bile, where it is enzymatically deconjugated by glucuronidases produced by gut bacteria and then further oxidized and reduced, forming stercobilin and urobilin that can be reabsorbed or excreted in the feces or urine (Wagner et al., 2015; Hamoud et al., 2018).

Since the gut represents a main location of bilirubin metabolism, a link between chronically increased UCB levels and gut health seems likely. We have recently reported on associations between UCB and colorectal cancer (CRC) risk in the European Prospective Investigation into Cancer and nutrition (EPIC) study, whereby serum UCB concentrations were positively associated with CRC risk in men and inversely associated in women (Seyed Khoei et al., 2020).
CRC is the third most common malignancy diagnosed and the fourth leading cause of cancer-related deaths worldwide (Arnold et al., 2017), and is expected to increase by a further $60 \%$ over the next decade. This increase is estimated to result in more than 2.2 million additional cases and 1.1 million annual deaths, by the year 2030 (Rawla et al., 2019).

Established CRC risk factors include high consumption of red/processed meat, low intake of dietary fibre, alcohol consumption, smoking, physical inactivity, obesity and height (World Cancer Research Fund/American Institute for Cancer Research, 2018). Increasingly, the gut microbiota has been implicated in CRC. Alterations in gut microbiota composition have been associated with a growing number of diseases, including cancer and particularly CRC (Schwabe and Jobin, 2013). More than $20 \%$ of the cancer burden worldwide is attributable to known infectious agents that are often normal residents of the intestinal microbiota (Zur Hausen, 2009).

Although a number of studies (Brennan and Garrett, 2016; Ternes et al., 2020) link certain members of the gut microbiota as causative factors in CRC development, the patho-etiological intricacies are poorly understood (Fong et al., 2020). Several mechanisms, including inflammation, bacterial pathogenicity, genotoxins and oxidative stress have been strongly implicated (Cheng et al., 2020), all of which have potential links to bilirubin metabolism.

Surprisingly, to date, potential associations between (increased) circulating UCB concentrations and the gut microbiota of adults remain unexplored. Therefore, this study aimed to evaluate whether (i) individuals with mildly elevated circulating UCB concentrations (i.e., GS) possess differences in their gut microbiota compared to age- and sex-matched controls, and to determine whether (ii) any observed effects are age- or sex-dependent.

\section{MATERIALS AND METHODS}

\section{Participants and Study Design}

The "BILIHEALTH" study was designed as an observational case-control study, at a single centre in Vienna, Austria as described more detailed previously (Mölzer et al., 2016; Mölzer et al., 2017).

Briefly, 128 healthy participants between 20 and 80 years of age were recruited from the general Austrian population. During the study, eight were excluded for medical reasons. Exclusion criteria included smoking, excess drinking, routine intake of medication and nutritional supplements, pregnancy, acute and chronic (inflammatory/metabolic) diseases, liver diseases, 
present or past neoplasia and organ transplants. Each participant completed an initial health check-up which covered fasting blood biochemistry including levels of UCB and liver enzymes, blood pressure, body weight/-height, and questionnaires.

A total of 80 males and 40 females completed the study. This sex distribution is representative of the occurrence of GS in the general population (Wallner et al., 2013a). All participants were age- and sex-matched, and study group allocation (GS vs Control, C) was based on the participants' fasting serum UCB concentrations $(</ \geq 17.1 \mu \mathrm{M})$ (Wallner et al., 2013a), as analysed by High-Performance Liquid Chromatography. Most of the GS participants showed visible signs of mild jaundice, observable by a yellowish pigmentation of the skin and the conjunctival membranes over the sclera. Liver parameters and parameters of haemolysis were within the normal ranges. Participants were furthermore allocated to age groups $(</ \geq 35$ years of age). In order to support diagnosis of GS, all participants of both study groups were required to fast the day before participating in the study, following a $400 \mathrm{kcal}$ fasting protocol (Radu and Atsmon, 2001; Wallner et al., 2013b). Furthermore, a complete overnight fast of $16 \pm 1$ hour was required before the day of blood sampling.

The study was approved by the Ethics Commission of the Medical University of Vienna (No. 1164/2014), was registered in ClinicalTrials.gov (NCT04792996) and was conducted in accordance with the Declaration of Helsinki. All participants provided signed informed consent prior to study participation.

\section{Faeces Sampling and Further Exclusion of Subjects}

Faecal samples were collected at home by the participants and stored in the refrigerator for not longer than overnight. Samples were handed over in the morning of the screening day at the General Hospital of Vienna, aliquoted to approximately $500 \mathrm{mg}$ in Eppendorf-tubes and stored at $-20^{\circ} \mathrm{C}$. Eight out of 128 participants were excluded due to exclusion criteria as mentioned above. In addition, 19 participants and their ageand sex-matched controls (in total 30) were excluded due to the lack of faecal sample or undetermined UGT1A1*28-genotype. Consequently, 90 age- and sex-matched participants, were considered for statistical analyses.

\section{DNA Extraction From Faeces}

DNA from faeces samples was extracted using a Phenol/ chloroform/isoamyl alcohol extraction protocol as previously described (Griffiths et al., 2000). After bead-beating and centrifugation, DNA was precipitated from the aqueous phase by adding 0.1 volume of $3 \mathrm{M}$ sodium acetate and 0.6 volumes of ice-cold isopropyl alcohol. DNA-pellets were rinsed with $70 \%$ ethanol and eluted in $100 \mu \mathrm{L}$ TlowE-buffer $(10 \mathrm{mM}$ Tris- $\mathrm{HCl} /$ $0.1 \mathrm{mM}$ EDTA dissolved in DEPC-treated water). DNA concentration and quality were determined using a NanoDrop 1000 Spectrometer including ND-1000 operation software set for nucleic acid DNA-50 (Thermo Fisher Scientific). The ratio of the absorbance at 260 and $280 \mathrm{~nm}$ (A260/280) was used to assess the purity of DNA and samples were diluted to $50 \mathrm{ng} / \mu \mathrm{L}$ with TlowE-buffer.

\section{Multiplex Polymerase Chain Reaction (Multiplex-PCR) for 16S rRNA Gene Amplicons}

A barcoding-multiplex tandem PCR (Berry et al., 2012) was performed targeting the V3-V4 region of the bacterial $16 \mathrm{~S}$ rRNA gene. Target and barcoding primers were designed as previously described (Hamady et al., 2008): HBact341F: 5' - CCTACGGGNGGCWGCAG-3' and HBact785R: 5'- GACTACHVGGGTATCTA-3'.

The reaction mix contained $2 \mu \mathrm{L}$ DNA (100 ng), 1x Taq buffer with $\mathrm{KCl}$ (B38), $0.2 \mathrm{mM}$ dNTPs, $2 \mathrm{mM} \mathrm{MgCl}_{2}, 1 \mu \mathrm{M}$ forward and reverse primer, $0.1 \mu \mathrm{g} / \mu \mathrm{L}$ BSA and $25 \mathrm{mU} / \mu \mathrm{L}$ recombinant Taq Polymerase (all from Thermo Fisher Scientific). In the two-step PCR, the first round was performed in triplicates with final volumes of $20 \mu \mathrm{L}$ per well and 25 cycles $\left(95^{\circ} \mathrm{C}\right.$ for $30 \mathrm{sec}$., $55^{\circ} \mathrm{C}$ for $30 \mathrm{sec}$. and $72^{\circ} \mathrm{C}$ for $60 \mathrm{sec}$.) and the second round was carried out with final volumes of $50 \mu \mathrm{L}$ by addition of reaction mix (Taq buffer, dNTPs, $\mathrm{MgCl}_{2}, \mathrm{BSA}$ and Polymerase as described before) with $5 \mu \mathrm{L}$ from first step pool, $2 \mu \mathrm{L}$ barcoding primer, and 5 cycles $\left(95^{\circ} \mathrm{C}\right.$ for $30 \mathrm{sec}$., $52^{\circ} \mathrm{C}$ for $30 \mathrm{sec}$., and $72^{\circ} \mathrm{C}$ for $60 \mathrm{sec}$.). Amplicons were purified using the Zymo Research (ZR-96) sequencing DNA Clean-up Kit (D4017) and eluted in $20 \mu \mathrm{L}$ per sample in PCR-grade water.

\section{Preparation and Sequencing}

The amplification performance was checked by electrophoresis: each sample ( $5 \mu \mathrm{L}$ with $1 \mu \mathrm{L}$ 6X DNA Gel Loading Dye (Thermo Fisher Scientific)) was loaded on $120 \mathrm{~mL} 1.5 \%$ agarose gel (Biozym, LE Agarose) in 1x TBE (89 mM Tris, $89 \mathrm{mM}$ boric acid, 2 mM EDTA) with $1.2 \mu \mathrm{L}$ GelRed and compared to a ladder (Thermo Fisher Scientific, $1 \mathrm{~kb}$ DNA Ladder, ready-to-use). Electrophoreses was set up with $80 \mathrm{~V}$ (BIO RAD, PowerPac Basic Power Supply) for approx. 60 minutes and analysed with a Biorad, Molecular Imager Gel Doc XR+ System with Image Lab Software for bands approximating 500 base pairs considering an amplicon length of 513 base pairs was expected.

Amplicons were quantified using the Quant-i PicoGreen dsDNA Assay Kit (Thermo Fisher Scientific) by comparison to a standard curve measured with an Infinite M200 Microplate Reader (Tecan Trading AG with i-control ${ }^{\mathrm{TM}}$ software).

An equimolar pool of $2 \times 10^{10}$ copies of amplicons per sample was prepared and sent to Mycrosynth AG (Balach, Switzerland) for sequencing on an Illumina MiSeq system.

\section{Bioinformatics}

A total number of 2840051 sequences were aligned (Herbold et al., 2015) using MOTHUR (Schloss et al., 2009) and QIIME (Caporaso et al., 2010) by the Division of Microbial Ecology, University of Vienna with an expected amplicon length of 513 base pairs based on a paired end read. Unique sequences (singletons) were removed and remaining sequences were sorted according their unique 8 nt barcode. 1348195 merged read pairs were assigned to 749 operational taxonomic units (OTUs) at species-level, identified using a 97\% identity threshold (Nguyen et al., 2016) and comparing to the SILVA database (Glockner et al., 2017). 
Sequence data has been deposited in the NCBI Sequence Read Archive under SRP316524.

\section{UCB Measurements Using High-Performance Liquid Chromatography (HPLC)}

Circulating UCB levels were measured in serum by HPLC following a well-established protocol (Wallner et al., 2013b; Seyed Khoei et al., 2020) using HPLC (HPLC, Merck, Hitachi, LaChrom, Vienna, Austria), equipped with a Fortis C18 HPLCcolumn $(4.6 \times 150 \mathrm{~mm}, 3 \mu \mathrm{m})$, a Phenomenex SecurityGuard ${ }^{\mathrm{TM}}$ cartridges for C18 HPLC-columns $(4 \times 3 \mathrm{~mm})$, and a photodiode array detector (PDA, Shimadzu). An isocratic mobile phase contained glacial acetic acid $(6.01 \mathrm{~g} / \mathrm{L})$ and $0.1 \mathrm{M} \mathrm{n}$ dioctylamine in HPLC grade methanol/water (96.5/3.5\%) was used. UCB was extracted from serum by mixing $40 \mu \mathrm{L}$ serum with $160 \mu \mathrm{L}$ mobile phase. After centrifugation, $50 \mu \mathrm{L}$ of the supernatant was injected at a flow rate of $1 \mathrm{~mL} / \mathrm{min}$.

\section{UGT1A1 Genotyping for TA Repeats in the UGT1A1*28 Promoter Region}

UGT1A1 alleles from whole blood were determined through melting curves, using QIAsymphony DSP DNA Midi Kits on a QIAsymphony SP automated system (QIAGEN). $10 \mu \mathrm{M}$ working solutions of LightCyclerFastStart DNA Master HybProbe Mix and primers were run on a LightCycler 480 Instrument II (Roche), as described previously by von Ahsen et al. (2000).

\section{Statistical Analyses}

Statistical analysis was performed using the computing environment $\mathrm{R}$ version 3.3.2 ( $\mathrm{R}$ Development Core Team, 2017). Additional packages used were vegan (Oksanen et al., 2007) for alpha-, beta-diversity-analysis and Adonis/ perMANOVA, cluster (Maechler et al., 2017) with wards method and indicspecies (Cáceres and Legendre, 2009) for indicator species analysis. Sequence data were subsampled for each sample to equal $95 \%$ of the counted sequences of the smallest sample size to avoid any bias from unequal sequencing depth. Generalized linear models were tested with the edgeR-package (McCarthy et al., 2012).

Normality was checked using the Shapiro-Wilk test in the coin-package of $\mathrm{R}$ (Hothorn et al., 2006). The non-parametric multivariate analyses of variance tests were performed by Adonis function which was set to 9999 permutations. Data are summarized according to their respective distribution. For parametric data, means \pm SD (standard deviation), for nonparametric variables, medians \pm IQR (inter-quartile range) are presented. For all statistical analyses, the significance level was based on P-value $\leq 0.05$.

\section{RESULTS}

\section{Characteristics of the Study Population}

Baseline characteristics of the study participants are presented in Table 1.
The GS group had significantly greater serum UCB levels and a reduced BMI (Table 1A), which was more pronounced in the older age group (above 35 years). This BMI difference between $\mathrm{C}$ and GS was more evident in females (Table 1B). The significant difference in UCB between groups was independent of sex (Tables 1A-C).

\section{Composition of the Gut Microbiota}

Isolation and sequencing were successful with Good's coverage between 98.5 - $99.7 \%$ (Table 2) and rarefaction curves showed asymptotic behaviour, indicating that sufficient sequencing depth had been achieved. 749 OTUs were identified prior to rarefaction. The Firmicutes/Bacteroidetes ratio, alpha and beta diversity were not significantly different between $\mathrm{C}$ vs. GS-groups (Table 2 and Figure 1), and PCoA ordination showed no distinct clusters (Figure 2)

Most abundant phyla (Figure 3A) in C and GS group were Firmicutes (C vs. GS: $68.4 \pm 12.2 \%$ vs. $67.6 \pm 11.5 \%, \mathrm{p} \sim 0.92$ ), Bacteroidetes $(16.8 \pm 10.8 \%$ vs. $18.4 \pm 10.6 \%, \mathrm{p} \sim 0.77)$, Actinobacteria $(12.3 \pm 8.9 \%$ vs. $10.9 \pm 10.4 \%, \mathrm{p} \sim 0.77)$, Proteobacteria $(1.2 \pm 1.7 \%$ vs. $1.8 \pm 3.9 \%, \mathrm{p} \sim 0.77)$, and Verrucomicrobia $(1.1 \pm 3.3 \%$ vs. $1.1 \pm 3.4 \%, \mathrm{p} \sim 0.99)$ (listed in decreasing order according C-group and abundance above 1\%). At a family level (Figure 3B) Lachnospiraceae (C vs. GS: $35.0 \pm$ $12.4 \%$ vs. $34.0 \pm 9.5 \%, \mathrm{p} \sim 0.77)$, Ruminococcaceae $(24.2 \pm 8.3 \%$ vs. $25.6 \pm 7.7 \%, \mathrm{p} \sim 0.77)$, Bifidobacteriaceae $(10.8 \pm 8.7 \%$ vs. $9.5 \pm 9.9 \%$, $\mathrm{p}$ $\sim 0.77)$, Bacteroidaceae $(10.4 \pm 8.2 \%$ vs. $12.4 \pm 8.5 \%, \mathrm{p} \sim 0.71)$, Prevotellaceae $(3.2 \pm 7.1 \%$ vs. $2.3 \pm 6.6 \%, \mathrm{p} \sim 0.77)$ and Erysipelotrichaceae $(2.7 \pm 3.0 \%$ vs. $1.6 \pm 1.7 \%, \mathrm{p} \sim 0.40)$, Veillonellaceae $(2.0 \pm 2.8 \%$ vs. $1.5 \pm 1.8 \%, \mathrm{p} \sim 0.72)$, Rikenellaceae $(1.0 \pm 0.9 \%$ vs. $1.5 \pm$ $1.6 \%, \mathrm{p} \sim 0.40)$, Coriobacteriaceae ( $1.6 \pm 1.4 \%$ vs. $1.4 \pm 1.1 \%, \mathrm{p} \sim 0.77$ ), Peptostreptococcaceae $(1.5 \pm 2.2 \%$ vs. $1.3 \pm 1.4 \%, \mathrm{p} \sim 0.72)$, Porphyromonadaceae $(1.3 \pm 3.1 \%$ vs. $1.3 \pm 1.3 \%, \mathrm{p} \sim 0.94)$, Verrucomicrobiaceae $(1.1 \pm 3.3 \%$ vs. $1.1 \pm 3.3 \%, \mathrm{p} \sim 0.99)$, Streptococcaceae $(1.0 \pm 1.4 \%$ vs. $1.0 \pm 1.4 \%, \mathrm{p} \sim 0.99)$, Enterobacteriaceae $(0.7 \pm 1.7 \%$ vs. $1.0 \pm 4.2 \%, \mathrm{p} \sim 0.77)$, Christensenellaceae $(0.6 \pm 09 \%$ vs. $1.0 \pm 1.4 \%, \mathrm{p} \sim 0.58)$ were predominant.

Abundances at a genus level (Figure 3C) were more diverse (listed in decreasing order according to C-group and abundance above 1\%): Faecalibacterium (C vs. GC: $12.5 \pm 7.6 \%$ vs. $12.8 \pm$ $5.3 \%, \mathrm{p} \sim 0.91)$, Blautia $(11.3 \pm 6.5 \%$ vs. $10.3 \pm 4.0 \%, \mathrm{p} \sim 0.71)$, Bifidobacterium $(10.8 \pm 8.7 \%$ vs. $9.5 \pm 9.9 \%, \mathrm{p} \sim 0.81)$, Bacteroides (10.4 $\pm 8.2 \%$ vs. $12.4 \pm 8.5 \%$, p 0.69), Incertae_Sedis (Lachnospiraceae) $(9.3 \pm 4.1 \%$ vs. $8.1 \pm 3.3 \%$, $\mathrm{p} \sim 0.69)$, Pseudobutyrivibri (5.5 $\pm 4.1 \%$ vs. $6.7 \pm 4.4 \%$, $\mathrm{p} \sim 0.69)$, Subdoligranulum $(3.3 \pm 2.4 \%$ vs. $3.4 \pm 2.8 \%, \mathrm{p} \sim 0.92)$, Prevotella (3.0 $\pm 7.0 \%$ vs. $2.1 \pm 6.6 \%, \mathrm{p} \sim 0.81)$, Anaerostipes $(3.1 \pm 2.9 \%$ vs. $2.8 \pm 2.4 \%, \mathrm{p} \sim 0.84)$, Ruminococcus $(3.0 \pm 2.9 \%$ vs. $4.2 \pm 3.3 \%$, $\mathrm{p} \sim 0.69)$, Incertae_Sedis (Ruminococcaceae) $(2.8 \pm 3.5 \%$ vs. $2.0 \pm$ $1.3 \%, \mathrm{p} \sim 0.69)$, Coprococcus $(1.7 \pm 1.5 \%$ vs. $1.7 \pm 1.6 \%, \mathrm{p} \sim 0.96)$, Dialister $(1.7 \pm 2.7 \%$ vs. $1.3 \pm 1.8 \%, \mathrm{p} \sim 0.72)$, Incertae_Sedis (Peptostreptococcaceae) $(1.5 \pm 2.2 \%$ vs. $1.3 \pm 1.4 \%, \mathrm{p} \sim 0.85)$, Dorea $(1.4 \pm 0.9 \%$ vs. $1.3 \pm 0.8 \%, \mathrm{p} \sim 0.91)$, Coprobacillus $(1.3 \pm$ $1.8 \%$ vs. $0.9 \pm 0.8 \%, \mathrm{p} \sim 0.69)$, Akkermansia $(1.1 \pm 3.3 \%$ vs. $1.1 \pm$ $3.3 \%, \mathrm{p} \sim 1.00)$, Incertae_Sedis (Erysipelotrichaceae) $(1.0 \pm 2.2 \%$ vs. $0.6 \pm 1.5 \%, \mathrm{p} \sim 0.69)$, Roseburia $(1.0 \pm 1.0 \%$ vs. $1.1 \pm 1.0 \%$, 
TABLE 1 | Demographic description of the study subjects (all subjects/females/males).

A. Baseline characteristics of Gilbert's Syndrome participants and their matched controls.
All subjects
p-value
1.00
0.91
$0.90 / 0.82$
$<0.001^{\star}$
$0.011^{*}$
$0.135 / 0.015^{\star}$

$31.0(18.5)$

$27 / 18$

$27.0(6.0) / 47.0(12.25)$

9.2 (3.4)

$3 / 21 / 21$

$23.9(6.2)$

$27.0(6.0) / 48.5(13.5)$

$32.6(9.4)$

$40 / 4 / 1$

$22.2(3.4)$

$21.5(2.8) / 22.8(4.5)$

B. Baseline characteristics of Gilbert's Syndrome participants and their matched controls among females.

Participants [n]

Median age $[\mathrm{yrs}]^{\Delta}$

Participants aged $\leq />35 \mathrm{yrs}[\mathrm{n} / \mathrm{n}]$

Age of participants $\leq />35 \mathrm{yrs}[\mathrm{yrs}]^{\Delta}$

UCB concentration $[\mu \mathrm{M}]^{\varnothing}$

UGT1A1*28 TA repeats [n 7/6_7/6]

BMI $\left[\mathrm{kg} / \mathrm{m}^{2}\right]^{\Delta}$

BMI $\left[\mathrm{kg} / \mathrm{m}^{2}\right] \leq />35 \mathrm{yrs}$

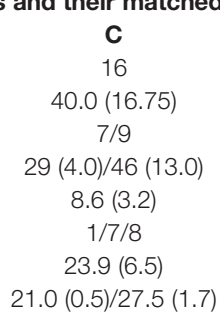

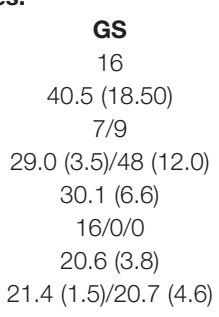

Female

p-value

1.000

0.949

1.000

$<0.001^{*}$

$0.024^{*}$

$0.549 / 0.012^{*}$

\begin{tabular}{|c|c|c|c|}
\hline \multicolumn{3}{|c|}{ C. Baseline characteristics of Gilbert's Syndrome participants and their matched controls among males. } & \multirow{2}{*}{$\begin{array}{c}\text { Male } \\
\text { p-value }\end{array}$} \\
\hline & C & GS & \\
\hline Participants [n] & 29 & 29 & 1.000 \\
\hline Median age $[y r s]^{\Delta}$ & $29.0(18.00)$ & $29.0(16.00)$ & 0.944 \\
\hline Age of participants $\leq 1>35 \mathrm{yrs}[\mathrm{yrs}]^{\Delta}$ & $26(6.0) / 49(10.0)$ & $26(5.5) / 51(12.0)$ & \\
\hline UCB concentration $[\mu \mathrm{M}]^{\varnothing}$ & $9.9(3.5)$ & $33.9(10.5)$ & $<0.001^{\star}$ \\
\hline UGT1A1*28 TA repeats $[n \text { 7/6_7/6] }]^{\square}$ & $2 / 14 / 13$ & $24 / 4 / 1$ & \\
\hline BMl $\left[\mathrm{kg} / \mathrm{m}^{2}\right]^{\Delta}$ & $23.9(4.9)$ & $22.4(2.4)$ & 0.101 \\
\hline
\end{tabular}

${ }^{\varnothing}$ Data are expressed as mean value $\pm S D .{ }^{\Delta}$ Data are expressed as median $\pm I Q R$. ${ }^{p}$-values $\leq 0.05$ show significant differences (t-test or Mann-Whitney-U-Statistic, respectively); Control (C) group and Gilbert's syndrome (GS) group ${ }^{\square}$ Insertion of additional TA repeats in the UGT1A1*28 promoter region; 7: Gilbert's syndrome, 6_7: heterozygous individuals, 6: wild type.

TABLE 2 | Medians sequencing data and diversity characteristics.

\begin{tabular}{|c|c|c|c|}
\hline & C & GS & $p$-value \\
\hline No. sequences & 13793 (3682) & 13647 (4821) & 0.6 \\
\hline Good's coverage & $0.994(0.0022)$ & $0.994(0.0020)$ & 0.9 \\
\hline No. observed OTUs & $159.6(29.8)$ & $163.4(27.2)$ & 0.2 \\
\hline Chao1 & 361.6 (78.2) & $360.0(87.8)$ & 0.9 \\
\hline Shannon & $3.438(0.4)$ & $3.520(0.5)$ & 0.2 \\
\hline inv. Simpson & $15.28(6.4)$ & $15.771(9.2)$ & 0.3 \\
\hline F/B-ratio & $4.52(6.8)$ & $4.25(4.5)$ & 0.5 \\
\hline
\end{tabular}

Medians \pm IQR are given. Mann-Whitney-U-statistic shows no significant difference between the control and Gilbert's syndrome-group. Control (C) group and Gillbert's syndrome (GS) group; OTU, Operational taxonomic units, F/B-ratio, Firmicutes/Bacteroidetes-ratio.

$\mathrm{p} \sim 0.87)$, Collinsella $(1.0 \pm 1.2 \%$ vs. $0.9 \pm 0.9 \%, \mathrm{p} \sim 0.84)$ Alistipes ( $0.9 \pm 0.9 \%$ vs. $1.5 \pm 1.6 \%, \mathrm{p} \sim 0.69)$, Streptococcus $(0.9$ $\pm 1.2 \%$ vs. $1.0 \pm 1.4 \%, \mathrm{p} \sim 0.91)$, Lachnospira $(0.9 \pm 1.15 \%$ vs. 1.1 $\pm 1.0 \%, \mathrm{p} \sim 0.71)$, Oscillibacter $(0.9 \pm 1.0 \%$ vs. $1.1 \pm 1.0 \%, \mathrm{p} \sim$ $0.69)$ and unclassified (Christensenellaceae) $(0.6 \pm 0.9 \%$ vs. $1.0 \pm$ $1.4 \%, \mathrm{p} \sim 0.91)$.

\section{Gut Microbiota and Bilirubin Phenotype}

Microbiota composition did not differ between the groups for all taxonomic levels and at OTU level based on the GS-phenotype and age $(</ \geq 35$ years). The factor sex was a significant determinant of microbial composition at genus level with greater observed diversity in females $(\mathrm{p}<0.05)$. Pairwise testing of the relative abundances of taxa on domain, phylum, class, family, genus and OTU-level showed no significant differences for GS-phenotype after adjusting for multiple testing with $\mathrm{BH}$ procedure (false discovery rate) with age, sex, and BMI as covariates using a generalized linear model.

An indicator species analysis (Table 3) resulted in very low abundance of OTUs and low association values $(\leq 0.512)$ with a strong dependency to the rarefaction step performed during subsampling (described in Statistical Analyses). This low association values indicate that there is little association between OTU relative abundances and phenotype. 
A

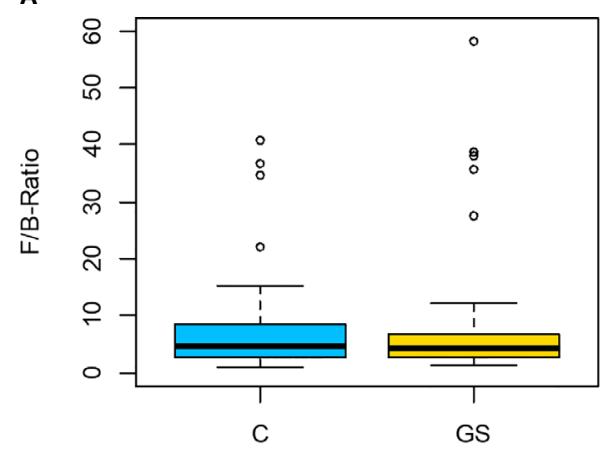

c

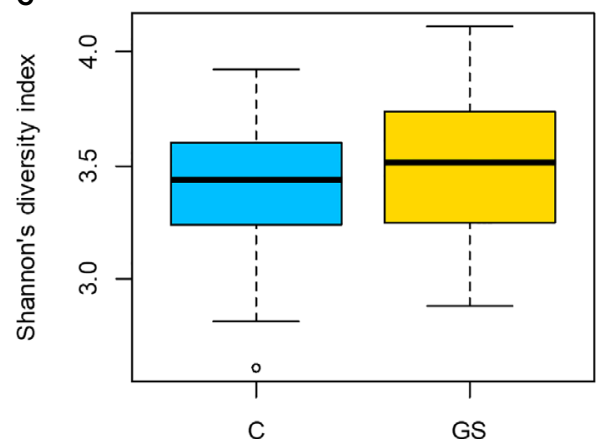

B

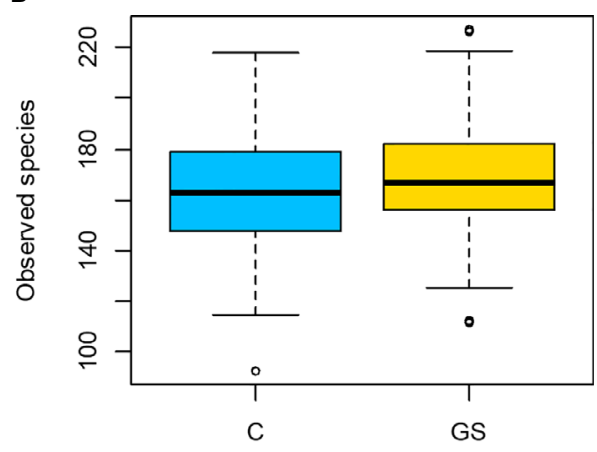

D

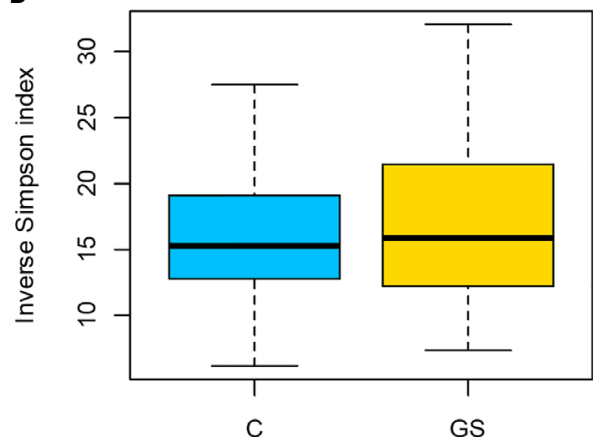

FIGURE 1 | Plots of diversity characteristics: (A) Firmicutes to Bacteroidetes ratio, (B) observed species, (C) Shannon's diversity index and (D) inv. Simpson index did not differ between the C/GS-groups ( $p>0.05)$. In $(\mathbf{A})$ participant B71 is not shown in C-group due to a F/B-ratio of 131.

\section{DISCUSSION}

Mild hyperbilirubinaemia (GS) with normal circulating liver transaminases, biliary markers, and red blood cell counts, is a benign condition that is highly prevalent among the general population. Elevated UCB levels are inversely associated with the risk of chronic diseases including some cancers. Since bilirubin is in part metabolised in the gut, we investigated whether participants with mildly increased UCB levels exhibit a different gut microbiota composition compared to age- and sex-matched controls. Such differences could help to better explain the link between lower CRC risk observed in GS individuals, but could also be linked to the lower risk for metabolic diseases.

In the present study, gut microbiota composition was determined using 16S rRNA gene-targeted sequencing, a popular approach to determine whether there are alterations in the microbiota linked to disease states (Rebolledo et al., 2017; Leiva-Gea et al., 2018; Das et al., 2021). Microbial patterns that are typically associated with proximal or distal CRC could not be detected in either group. Compared to controls no differences between alpha and beta diversity and no over- and underrepresentation of genera were detected in GS individuals.
The bacterial community in both groups was dominated by the typical phyla Firmicutes and Bacteroidetes. A difference in the percentage of Firmicutes and Proteobacteria, which have been associated with the mucosa of CRC patients (Gao et al., 2015) was not detected. Similarly, no difference was found in the ranks of family and genus. While the lack of a statistically significant association between the microbiota and GS could be due to cohort size, similar-sized studies have found differences in the microbiota within disease states (Rebolledo et al., 2017; LeivaGea et al., 2018; Das et al., 2021; Shuntian et al., 2021). As this is a reasonable cohort size for a pilot study, we conclude that if the microbiota is affected by GS, it must be a relatively minor effect on community composition.

In the literature, only four bacteria (Bacteroides fragilis, Clostridium ramosum, Clostridium perfringens, and Clostridioides difficile) have been linked to bilirubin metabolism so far, since they were able to reduce urobilinogen mixtures, including half-stercobilinogen and stercobilin, under in vitro and in vivo conditions (Chen and Yuan, 2020). These species are all common members of the intestinal microbiota (Vitek et al., 2005; Hamoud et al., 2018).

Although microbiota data of GS have not been published previously, there are a small number of reports from newborns 
A

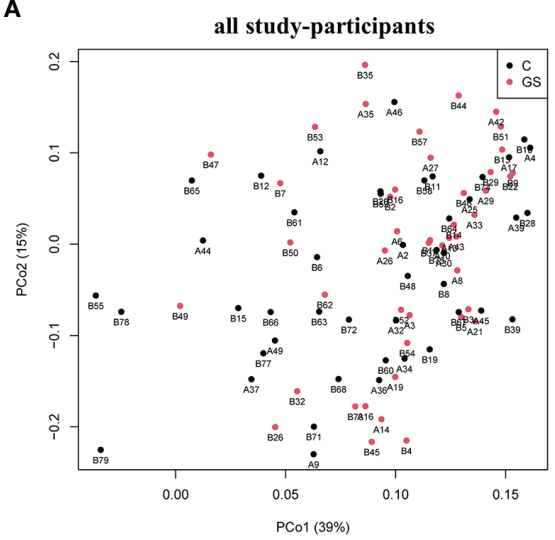

B

B

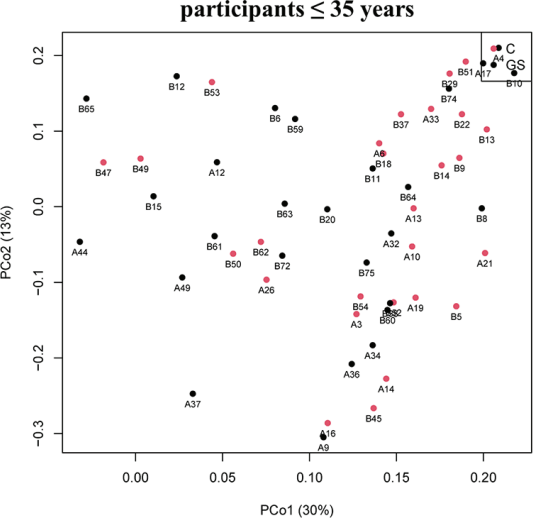

C

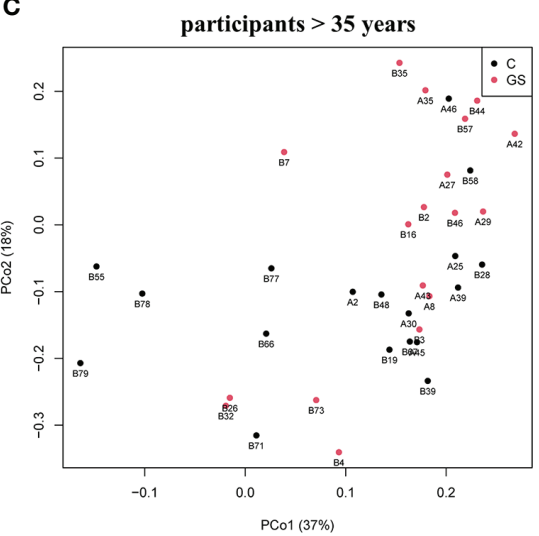

FIGURE 2 | Plots of Principal coordinates analysis based on Bray-Curtis dissimilarity matrix for rarefied OTUs of C and GS samples and phenotype for (A) all studyparticipants and separated for age $\mathbf{( B )} \leq 35$ years and $\mathbf{( C )}>35$ years. No distinct clusters are distinguishable.

A

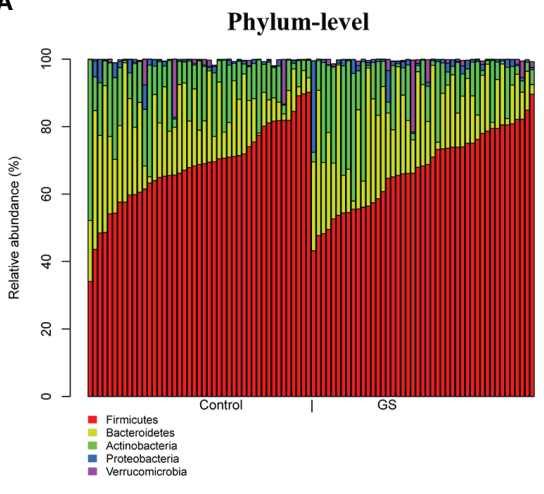

B

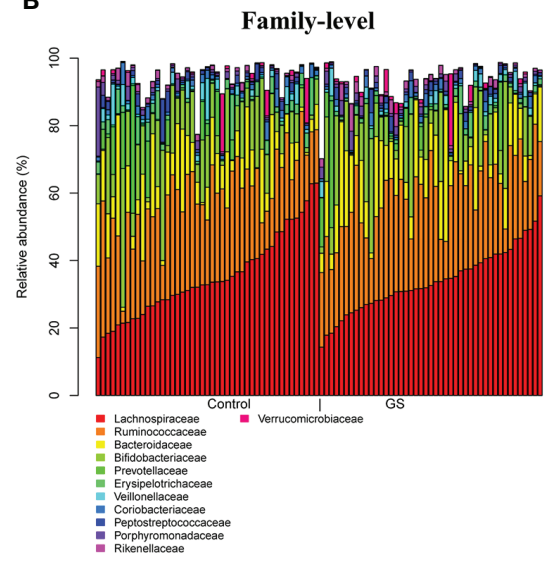

C

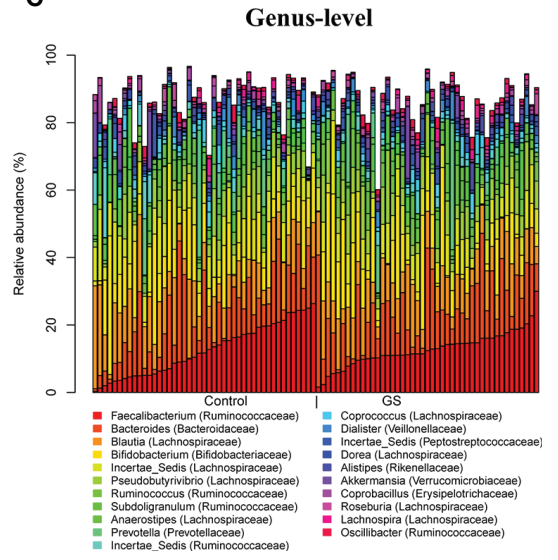

FIGURE 3 | Plots of taxonomic profiles for (A) phylum-level, (B) family-level and (C) genus-level from BiliHealth-gut-samples for each group (Controls and Gilbert's Syndrome). Abundant taxa with a mean relative abundance $>1 \%$ are shown. There are no significant differences between $\mathrm{C} \&$ GS group on all taxonomic levels.

with jaundice, which experience much higher and likely pathogenic bilirubin concentrations in the blood, when compared to GS. In jaundiced neonates, Clostridium perfringens was significantly elevated, which was considered as feedback on the severe hyperbilirubinemic conditions in the neonates (Dong et al., 2018). Further, in vitro data show that bilirubin is protective to the bacterial pathogen Escherichia coli O157:H7, but highly toxic to the bacterium Enteroccocus faecalis (Nobles et al., 2013).

Due to non-robust results of our OTU analysis, we could not reliably identify indicator taxa. Intriguingly, no OTUs classified to the above-mentioned bacteria were identified as indicators for GS in this cohort. Regardless of the latter, OTU_295 with a sequence identity of $90 \%$ (366/407 nucleotides with 4 gaps) was identified with the highest association value (0.521) that can be assigned to the GS-group. A subsequent BLAST (Zhang et al., 2000) analysis identified Christensenella minuta strain YIT 12065
(Morotomi et al., 2012) as the species closest to the sequence of OTU_295. Indeed, Goodrich et al. (2014) had previously associated this species with lean body mass. As GS individuals have a comparably lower BMI (Seyed Khoei et al., 2018) this result could be a first link to a lower CRC and potentially CVD risk.

Both study groups were generally very healthy, which could have masked potential microbial patterns. Sex was the only factor contributing to a slight difference in the composition of the genus-level microbiota profile, which has also been reported in other studies (Kim et al., 2020) but the lack of other associations could also be due to confounding factors in our cohort such as diet, race, medications or BMI.

Bile plays an important part in bilirubin metabolism. Upon conjugation, multidrug resistance-associated protein 2 transports conjugated bilirubin into the duodenum via the biliary tract and passes through the small intestines until it 
TABLE 3 | Summary of an indicator species analysis.

\begin{tabular}{|c|c|c|c|c|c|c|c|c|c|}
\hline & \multirow[t]{2}{*}{ Assoc. } & \multirow[t]{2}{*}{ p-value } & \multirow[t]{2}{*}{ Closest related species } & \multirow[t]{2}{*}{ Accession } & \multirow[t]{2}{*}{ Identity [\%] } & \multicolumn{2}{|c|}{ C } & \multicolumn{2}{|c|}{ GS } \\
\hline & & & & & & \multicolumn{2}{|c|}{$\begin{array}{c}\text { Mean abundance \& sd } \\
{[\%]}\end{array}$} & \multicolumn{2}{|c|}{$\begin{array}{c}\text { Mean abundance \& sd } \\
{[\%]}\end{array}$} \\
\hline \multicolumn{10}{|l|}{ C-group } \\
\hline OTU_200 & 0.509 & $0.032^{*}$ & Emergencia timonensis strain SN18 & NR_144737.1 & 89 & 5.8E-02 & 8.6E-02 & $2.0 \mathrm{E}-02$ & 3.3E-02 \\
\hline OTU_258 & 0.486 & $0.016^{\star}$ & Acetanaerobacterium elongatum strain $Z 7$ & NR_042930.1 & 90 & $1.9 \mathrm{E}-02$ & 3.7E-02 & 1.1E-02 & $2.1 \mathrm{E}-02$ \\
\hline OTU_230 & 0.394 & $0.015^{\star}$ & Mitsuokella jalaludinii strain M9 & NR_028840.1 & 99 & $1.5 \mathrm{E}-01$ & $5.2 \mathrm{E}-01$ & 2.3E-03 & 7.4E-03 \\
\hline OTU_319 & 0.356 & $0.034^{*}$ & Olsenella scatoligenes strain SK9K4 & NR_134781.1 & 94 & 5.0E-02 & 1.6E-01 & 4.5E-03 & 1.6E-02 \\
\hline \multicolumn{10}{|l|}{ GS-Group } \\
\hline OTU_295 & 0.521 & $0.025^{\star}$ & Christensenella minuta strain YIT 12065 & NR_112900.1 & 90 & 2.6E-02 & 5.6E-02 & 4.6E-02 & 5.9E-02 \\
\hline OTU_392 & 0.509 & $0.006^{\star \star}$ & Prevotella intermedia strain B422 & NR_026119.1 & 99 & 1.3E-02 & 1.9E-02 & $2.0 \mathrm{E}-02$ & 2.3E-02 \\
\hline OTU_465 & 0.464 & $0.01^{\star \star}$ & Aggregatibacter aphrophilus strain CIP 70.73 & NR_116167.1 & 99 & 1.4E-02 & 1.9E-02 & 1.6E-02 & 2.0E-02 \\
\hline OTU_113 & 0.456 & $0.026^{\star}$ & Rhodospirillum rubrum strain ATCC 11170 & NR_074249.1 & 87 & 2.0E-02 & 7.2E-02 & $2.4 \mathrm{E}-01$ & 8.8E-01 \\
\hline OTU_109 & 0.438 & $0.022^{*}$ & Novispirillum itersonii strain NBRC 15648 & NR_113793.1 & 87 & 4.4E-02 & $2.5 \mathrm{E}-01$ & 2.0E-01 & 5.7E-01 \\
\hline OTU_163 & 0.418 & $0.01^{\star \star}$ & Kiloniella majae strain M56.1 & NR_152635.1 & 87 & $3.4 \mathrm{E}-03$ & $8.8 \mathrm{E}-03$ & $1.1 \mathrm{E}-01$ & 3.9E-01 \\
\hline OTU_139 & 0.393 & $0.034^{*}$ & Bacteroides coprophilus strain CB42 & NR_041461.1 & 99 & 5.1E-03 & 2.7E-02 & 4.9E-01 & $2.1 \mathrm{E}+00$ \\
\hline OTU_391 & 0.379 & $0.033^{*}$ & Spiroplasma alleghenense strain PLHS-1 & NR_025697.1 & 85 & $2.8 \mathrm{E}-03$ & $9.8 \mathrm{E}-03$ & 3.6E-02 & 9.9E-02 \\
\hline OTU_263 & 0.378 & $0.037^{\star}$ & Ethanoligenens harbinense strain YUAN-3 & NR_074333.1 & 92 & 1.3E-02 & $4.2 \mathrm{E}-02$ & 2.7E-02 & 7.6E-02 \\
\hline OTU_471 & 0.378 & $0.031^{*}$ & Parabacteroides distasonis strain ATCC 8503 & NR_074376.1 & 98 & 8.5E-03 & 4.3E-02 & 1.5E-02 & 4.6E-02 \\
\hline
\end{tabular}

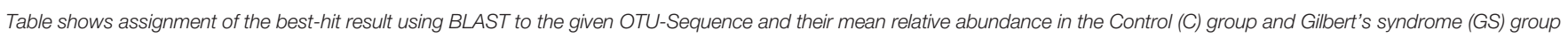

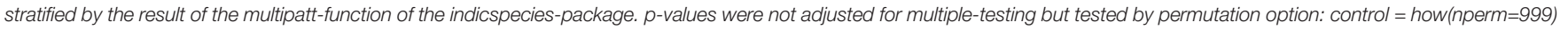
${ }^{*} p$-values $\leq 0.05$ and ${ }^{* *} p$-values $\leq 0.01$.

reaches the distal ileum and colon. Conjugated bilirubin is then again deconjugated by bacterial $\beta$-glucuronidases. Mainly in the large intestine, the intestinal microbiota metabolise UCB to urobilin and stercobilin. However, part of the deconjugated bilirubin of the bile is reabsorbed as part of the enterohepatic circulation before reaching the rectum (Bulmer et al., 2011; Chen and Yuan, 2020). Bile acids in bile are also secreted in the intestinal lumen and are subsequently re-absorbed in the terminal ileum and transported back to the liver for recycling. Some bile acids, however, reach the colon and are modified by the gut microbiota, which affects their physicochemical properties as well as inhibitory activity on bacteria. Therefore, bile acids also shape the composition and function of the intestinal microbiota. While there is no data available about a potential differences in bile acid composition within GS subjects, data from a genome wide analysis show that the UGT1A1 GS SNP variant rs6742078 is associated with gallstone disease in men (Buch et al., 2010), which might also affect gut microbiota composition. More data are needed in future to explore this question and to better understand the interplay between bile acids, bile pigments and microbiota composition.

We were not able to analyse UCB and stercobilin in the faeces of the subjects. Concentrations of both metabolites should be complementary, considering previous experiments demonstrating an increase in DNA strand breaks in human cancer cells depending on the concentration of these bile pigments (Mölzer et al., 2013b). Further, mutagenesis induced by the food-borne mutagen aflatoxin B1 was abrogated by both urobilin and stercobilin in the AMES Test. These findings point towards the importance of these compounds in gut metabolism and the interplay with food derived mutagens, which play a role in CRC development (Mölzer et al., 2013a).

In conclusion, this study indicates that, in the absence of acute inflammation or neoplasia, mildly elevated chronic UCB concentration in the blood in GS, which is associated with improved metabolic health, is not associated with an altered gut microbial composition when compared to a healthy age- and sex- matched control group.

\section{DATA AVAILABILITY STATEMENT}

Sequence data has been deposited in the NCBI Sequence Read Archive under SRP316524.

\section{ETHICS STATEMENT}

The studies involving human participants were reviewed and approved by Ethics Commission of the Medical University of Vienna. The patients/participants provided their written informed consent to participate in this study.

\section{AUTHOR CONTRIBUTIONS}

$\mathrm{K}-\mathrm{HW}, \mathrm{CM}$, and $\mathrm{MH}-\mathrm{W}$ designed the study and collected data. PZ, CM, and MH-W performed the analyses. PZ and $\mathrm{K}-\mathrm{HW}$ drafted the manuscript with input from all authors. 
PZ performed formal analyses and provided all figures and tables. All co-authors supported in interpreting the results. KHW and DB supervised the project covering different fields of scientific expertise. All authors contributed to the article and approved the submitted version.

\section{FUNDING}

PZ was funded by the FWF Stand-Alone Project P 29608 and supported by the Vienna Doctoral School of Pharmaceutical,

\section{REFERENCES}

Arnold, M., Sierra, M. S., Laversanne, M., Soerjomataram, I., Jemal, A., and Bray, F. (2017). Global Patterns and Trends in Colorectal Cancer Incidence and Mortality. Gut 66 (4), 683-691. doi: 10.1136/gutjnl-2015-310912

Berry, D., Ben Mahfoudh, K., Wagner, M., and Loy, A. (2012). Barcoded Primers Used in Multiplex Amplicon Pyrosequencing Bias Amplification (Vol 77, Pg 7846, 2011). Appl. Environ. Microbiol. 78 (2), 612-612. doi: 10.1128/ Aem.07448-11

Brennan, C. A., and Garrett, W. S. (2016). Gut Microbiota, Inflammation, and Colorectal Cancer. Annu. Rev. Microbiol. 70, 395-411. doi: 10.1146/annurevmicro-102215-095513

Buch, S., Schafmayer, C., Volzke, H., Seeger, M., Miquel, J. F., Sookoian, S. C., et al. (2010). Loci From a Genome-Wide Analysis of Bilirubin Levels Are Associated With Gallstone Risk and Composition. Gastroenterology 139 (6), 1942-1951 e1942. doi: 10.1053/j.gastro.2010.09.003

Bulmer, A. C., Bakrania, B., Du Toit, E. F., Boon, A. C., Clark, P. J., Powell, L. W., et al. (2018). Bilirubin Acts as a Multipotent Guardian of Cardiovascular Integrity: More Than Just a Radical Idea. Am. J. Physiol. Heart Circ. Physiol. 315 (3), H429-H447. doi: 10.1152/ajpheart.00417.2017

Bulmer, A. C., Coombes, J. S., Blanchfield, J. T., Toth, I., Fassett, R. G., and Taylor, S. M. (2011). Bile Pigment Pharmacokinetics and Absorption in the Rat: Therapeutic Potential for Enteral Administration. Br. J. Pharmacol. 164 (7), 1857-1870. doi: 10.1111/j.1476-5381.2011.01413.x

Bulmer, A. C., Ried, K., Blanchfield, J. T., and Wagner, K. H. (2008). The AntiMutagenic Properties of Bile Pigments. Mutat. Res. 658 (1-2), 28-41. doi: 10.1016/j.mrrev.2007.05.001

Bulmer, A. C., Verkade, H. J., and Wagner, K. H. (2013). Bilirubin and Beyond: A Review of Lipid Status in Gilbert's Syndrome and its Relevance to Cardiovascular Disease Protection. Prog. Lipid Res. 52 (2), 193-205. doi: 10.1016/j.plipres.2012.11.001

Cáceres, M. D., and Legendre, P. (2009). Associations Between Species and Groups of Sites: Indices and Statistical Inference. Ecology 90 (12), 3566-3574. doi: $10.1890 / 08-1823.1$

Caporaso, J. G., Kuczynski, J., Stombaugh, J., Bittinger, K., Bushman, F. D., Costello, E. K., et al. (2010). QIIME Allows Analysis of High-Throughput Community Sequencing Data. Nat. Methods 7 (5), 335-336. doi: 10.1038/nmeth.f.303

Cheng, Y., Ling, Z., and Li, L. (2020). The Intestinal Microbiota and Colorectal Cancer. Front. Immunol. 11, 615056. doi: 10.3389/fimmu.2020.615056

Chen, K., and Yuan, T. (2020). The Role of Microbiota in Neonatal Hyperbilirubinemia. Am. J. Transl. Res. 12 (11), 7459-7474.

Development Core Team, R. (2017). "R: A Language and Environment for Statistical Computing" (Vienna, Austria: R Foundation for Statistical Computing).

Dong, T., Chen, T., White, R. A.3rd, Wang, X., Hu, W., Liang, Y., et al. (2018). Meconium Microbiome Associates With the Development of Neonatal Jaundice. Clin. Transl. Gastroenterol. 9 (9), 182. doi: 10.1038/s41424-018-0048-x

Fong, W., Li, Q., and Yu, J. (2020). Gut Microbiota Modulation: A Novel Strategy for Prevention and Treatment of Colorectal Cancer. Oncogene 39 (26), 49254943. doi: 10.1038/s41388-020-1341-1

Gao, Z. G., Guo, B. M., Gao, R. Y., Zhu, Q. C., and Qin, H. L. (2015). Microbiota Disbiosis is Associated With Colorectal Cancer. Front. Microbiol. 6, 20. doi: $10.3389 /$ fmicb. 2015.00020
Nutritional and Sport Sciences' Completion Grant of the University of Vienna.

\section{ACKNOWLEDGMENTS}

The authors thank the entire project team for their effort and their important contributions throughout the project, specifically Dr. Alessandra Riva and Benjamin Zwirzitz, MSc from the Department of Microbiology and Ecosystem Science on amplicon sequence analysis.

Glockner, F. O., Yilmaz, P., Quast, C., Gerken, J., Beccati, A., Ciuprina, A., et al. (2017). 25 Years of Serving the Community With Ribosomal RNA Gene Reference Databases and Tools. J. Biotechnol. 261, 169-176. doi: 10.1016/ j.jbiotec.2017.06.1198

Goodrich, J. K., Waters, J. L., Poole, A. C., Sutter, J. L., Koren, O., Blekhman, R., et al. (2014). Human Genetics Shape the Gut Microbiome. Cell 159 (4), 789799. doi: 10.1016/j.cell.2014.09.053

Griffiths, R. I., Whiteley, A. S., O’Donnell, A. G., and Bailey, M. J. (2000). Rapid Method for Coextraction of DNA and RNA From Natural Environments for Analysis of Ribosomal DNA- and rRNA-Based Microbial Community Composition. Appl. Environ. Microbiol. 66 (12), 5488-5491. doi: 10.1128/ AEM.66.12.5488-5491.2000

Hamady, M., Walker, J. J., Harris, J. K., Gold, N. J., and Knight, R. (2008). ErrorCorrecting Barcoded Primers for Pyrosequencing Hundreds of Samples in Multiplex. Nat. Methods 5 (3), 235-237. doi: 10.1038/nmeth.1184

Hamoud, A. R., Weaver, L., Stec, D. E., and Hinds, T. D.Jr. (2018). Bilirubin in the Liver-Gut Signaling Axis. Trends Endocrinol. Metab. 29 (3), 140-150. doi: 10.1016/j.tem.2018.01.002

Herbold, C. W., Pelikan, C., Kuzyk, O., Hausmann, B., Angel, R., Berry, D., et al. (2015). A Flexible and Economical Barcoding Approach for Highly Multiplexed Amplicon Sequencing of Diverse Target Genes. Front. Microbiol. 6, 731. doi: 10.3389/fmicb.2015.00731

Hothorn, T., Hornik, K., van de Wiel, M. A., and Zeileis, A. (2006). A Lego System for Conditional Inference. Am. Statistician 60 (3), 257-263. doi: 10.1198/ 000313006X118430

Kim, Y. S., Unno, T., Kim, B. Y., and Park, M. S. (2020). Sex Differences in Gut Microbiota. World J. Mens Health 38 (1), 48-60. doi: 10.5534/wjmh.190009

Kwon, Y. J., Lee, Y. J., Park, B. J., Hong, K. W., and Jung, D. H. (2018). Total Serum Bilirubin and 8-Year Incident Type 2 Diabetes Mellitus: The Korean Genome and Epidemiology Study. Diabetes Metab. 44 (4), 346-353. doi: 10.1016/ j.diabet.2017.07.004

Leiva-Gea, I., Sanchez-Alcoholado, L., Martin-Tejedor, B., Castellano-Castillo, D., Moreno-Indias, I., Urda-Cardona, A., et al. (2018). Gut Microbiota Differs in Composition and Functionality Between Children With Type 1 Diabetes and MODY2 and Healthy Control Subjects: A Case-Control Study. Diabetes Care 41 (11), 2385-2395. doi: 10.2337/dc18-0253

Maechler, M., Rousseeuw, P., Struyf, A., Hubert, M., and Hornik, K. (2017). "Finding Groups in Data': Cluster Analysis Extended Rousseeuw Et Al.”. 2.0.6 Ed.). Available at: https://stat.ethz.ch/R-manual/Rdevel/library/cluster/ DESCRIPTION

McCarthy, D. J., Chen, Y., and Smyth, G. K. (2012). Differential Expression Analysis of Multifactor RNA-Seq Experiments With Respect to Biological Variation. Nucleic Acids Res. 40 (10), 4288-4297. doi: 10.1093/nar/ gks042

Mölzer, C., Huber, H., Steyrer, A., Ziesel, G. V., Wallner, M., Hong, H. T., et al. (2013a). Bilirubin and Related Tetrapyrroles Inhibit Food-Borne Mutagenesis: A Mechanism for Antigenotoxic Action Against a Model Epoxide. J. Nat. Prod. 76 (10), 1958-1965. doi: 10.1021/np4005807

Mölzer, C., Pfleger, B., Putz, E., Rossmann, A., Schwarz, U., Wallner, M., et al. (2013b). In Vitro DNA-Damaging Effects of Intestinal and Related Tetrapyrroles in Human Cancer Cells. Exp. Cell Res. 319 (4), 536-545. doi: 10.1016/j.yexcr.2012.12.003 
Mölzer, C., Wallner, M., Kern, C., Tosevska, A., Schwarz, U., Zadnikar, R., et al. (2016). Features of an Altered AMPK Metabolic Pathway in Gilbert's Syndrome, and its Role in Metabolic Health. Sci. Rep. 6, 30051. doi: 10.1038/ srep30051

Mölzer, C., Wallner, M., Kern, C., Tosevska, A., Zadnikar, R., Doberer, D., et al. (2017). Characteristics of the Heme Catabolic Pathway in Mild Unconjugated Hyperbilirubinemia and Their Associations With Inflammation and Disease Prevention. Sci. Rep. 7 (1), 755. doi: 10.1038/s41598-017-00933-y

Morotomi, M., Nagai, F., and Watanabe, Y. (2012). Description of Christensenella Minuta Gen. Nov., Sp. Nov., Isolated From Human Faeces, Which Forms a Distinct Branch in the Order Clostridiales, and Proposal of Christensenellaceae Fam. Nov. Int. J. Syst. Evol. Microbiol. 62 (Pt 1), 144-149. doi: 10.1099/ ijs.0.026989-0

Nguyen, N. P., Warnow, T., Pop, M., and White, B. (2016). A Perspective on 16S rRNA Operational Taxonomic Unit Clustering Using Sequence Similarity. NPJ Biofilms Microbiomes 2, 16004. doi: 10.1038/npjbiofilms.2016.4

Nobles, C. L., Green, S. I., and Maresso, A. W. (2013). A Product of Heme Catabolism Modulates Bacterial Function and Survival. PloS Pathog. 9 (7), e1003507. doi: 10.1371/journal.ppat.1003507

Oksanen, J., Kindt, R., Legendre, P., O’Hara, B., Stevens, M. H. H., Oksanen, M. J., et al. (2007). The Vegan Package. Community Ecol. Package 10, 631-637.

Radu, P., and Atsmon, J. (2001). Gilbert's Syndrome-Clinical and Pharmacological Implications. Isr. Med. Assoc. J. 3 (8), 593-598.

Rawla, P., Sunkara, T., and Barsouk, A. (2019). Epidemiology of Colorectal Cancer: Incidence, Mortality, Survival, and Risk Factors. Prz Gastroenterol. 14 (2), 89-103. doi: 10.5114/pg.2018.81072

Rebolledo, C., Cuevas, A., Zambrano, T., Acuña, J. J., Jorquera, M. A., Saavedra, K., et al. (2017). Bacterial Community Profile of the Gut Microbiota Differs Between Hypercholesterolemic Subjects and Controls. BioMed. Res. Int. 2017, 8127814. doi: 10.1155/2017/8127814

Schloss, P. D., Westcott, S. L., Ryabin, T., Hall, J. R., Hartmann, M., Hollister, E. B., et al. (2009). Introducing Mothur: Open-Source, Platform-Independent, Community-Supported Software for Describing and Comparing Microbial Communities. Appl. Environ. Microbiol. 75 (23), 7537-7541. doi: 10.1128/ AEM.01541-09

Schwabe, R. F., and Jobin, C. (2013). The Microbiome and Cancer. Nat. Rev. Cancer 13 (11), 800-812. doi: 10.1038/nrc3610

Seyed Khoei, N., Grindel, A., Wallner, M., Molzer, C., Doberer, D., Marculescu, R., et al. (2018). Mild Hyperbilirubinaemia as an Endogenous Mitigator of Overweight and Obesity: Implications for Improved Metabolic Health. Atherosclerosis 269, 306-311. doi: 10.1016/j.atherosclerosis.2017.12.021

Seyed Khoei, N., Jenab, M., Murphy, N., Banbury, B. L., Carreras-Torres, R., Viallon, V., et al. (2020). Circulating Bilirubin Levels and Risk of Colorectal Cancer: Serological and Mendelian Randomization Analyses. BMC Med. 18 (1), 229. doi: 10.1186/s12916-020-01703-w

Shuntian, C., Yanyun, F., Bangzhou, Z., Jinzhou, L., Xiaoning, Y., Yunpeng, L., et al. (2021). Appendectomy Is Associated With Alteration of Human Gut Bacterial and Fungal Communities. Front. Microbiol. doi: 10.3389/ fmicb.2021.724980

Stocker, R. (2004). Antioxidant Activities of Bile Pigments. Antioxid Redox Signal 6 (5), 841-849. doi: 10.1089/ars.2004.6.841

Ternes, D., Karta, J., Tsenkova, M., Wilmes, P., Haan, S., and Letellier, E. (2020). Microbiome in Colorectal Cancer: How to Get From Meta-Omics to Mechanism? Trends Microbiol. 28 (5), 401-423. doi: 10.1016/j.tim. 2020.01 .001

Vitek, L., and Tiribelli, C. (2020). Bilirubin, Intestinal Integrity, the Microbiome, and Inflammation. N. Engl. J. Med. 383 (7), 684-686. doi: 10.1056/ NEJMcibr2013250
Vitek, L., Zelenka, J., Zadinova, M., and Malina, J. (2005). The Impact of Intestinal Microflora on Serum Bilirubin Levels. J. Hepatol. 42 (2), 238-243. doi: 10.1016/ j.jhep.2004.10.012

von Ahsen, N., Oellerich, M., and Schutz, E. (2000). DNA Base Bulge vs Unmatched End Formation in Probe-Based Diagnostic Insertion/Deletion Genotyping: Genotyping the UGT1A1 (TA)(n) Polymorphism by Real-Time Fluorescence PCR. Clin. Chem. 46 (12), 1939-1945. doi: 10.1093/clinchem/46.12.1939

Wagner, K. H., Shiels, R. G., Lang, C. A., Seyed Khoei, N., and Bulmer, A. C. (2018). Diagnostic Criteria and Contributors to Gilbert's Syndrome. Crit. Rev. Clin. Lab. Sci. 55 (2), 129-139. doi: 10.1080/10408363.2018.1428526

Wagner, K. H., Wallner, M., Molzer, C., Gazzin, S., Bulmer, A. C., Tiribelli, C., et al. (2015). Looking to the Horizon: The Role of Bilirubin in the Development and Prevention of Age-Related Chronic Diseases. Clin. Sci. (Lond) 129 (1), 125. doi: $10.1042 / \operatorname{cs} 20140566$

Wallner, M., Antl, N., Rittmannsberger, B., Schreidl, S., Najafi, K., Mullner, E., et al. (2013a). Anti-Genotoxic Potential of Bilirubin In Vivo: Damage to DNA in Hyperbilirubinemic Human and Animal Models. Cancer Prev. Res. (Phila) 6 (10), 1056-1063. doi: 10.1158/1940-6207.Capr-13-0125

Wallner, M., Bulmer, A. C., Molzer, C., Mullner, E., Marculescu, R., Doberer, D., et al. (2013b). Haem Catabolism: A Novel Modulator of Inflammation in Gilbert's Syndrome. Eur. J. Clin. Invest. 43 (9), 912-919. doi: 10.1111/eci.12120

Wallner, M., Marculescu, R., Doberer, D., Wolzt, M., Wagner, O., Vitek, L., et al. (2013c). Protection From Age-Related Increase in Lipid Biomarkers and Inflammation Contributes to Cardiovascular Protection in Gilbert's Syndrome. Clin. Sci. (Lond) 125 (5), 257-264. doi: 10.1042/CS20120661

World Cancer Research Fund/American Institute for Cancer Research (2018). "Continuous Update Project Expert Report 2018. Diet, Nutrition, Physical Activity and Cancer".

Das, T., Jayasudha, R., Chakravarthy, A., Prashanthi, G. S., Bhargava, A., Tyagi, M., et al (2021). Alterations in the Gut Bacterial Microbiome in People With Type 2 Diabetes Mellitus and Diabetic Retinopathy. Sci. Rep. 11, 2738 doi: 10.1038/s41598-021-82538-0

Zhang, Z., Schwartz, S., Wagner, L., and Miller, W. (2000). A Greedy Algorithm for Aligning DNA Sequences. J. Comput. Biol. 7 (1-2), 203-214. doi: 10.1089/ 10665270050081478

Zucker, S. D., Horn, P. S., and Sherman, K. E. (2004). Serum Bilirubin Levels in the U.S. Population: Gender Effect and Inverse Correlation With Colorectal Cancer. Hepatology 40 (4), 827-835. doi: 10.1002/hep.20407

Zur Hausen, H. (2009). The Search for Infectious Causes of Human Cancers: Where and Why. Virology 392 (1), 1-10. doi: 10.1016/j.virol.2009.06.001

Conflict of Interest: The authors declare that the research was conducted in the absence of any commercial or financial relationships that could be construed as a potential conflict of interest.

Publisher's Note: All claims expressed in this article are solely those of the authors and do not necessarily represent those of their affiliated organizations, or those of the publisher, the editors and the reviewers. Any product that may be evaluated in this article, or claim that may be made by its manufacturer, is not guaranteed or endorsed by the publisher.

Copyright (C) 2021 Zöhrer, Hana, Seyed Khoei, Mölzer, Hörmann-Wallner, Tosevska, Doberer, Marculescu, Bulmer, Herbold, Berry and Wagner. This is an open-access article distributed under the terms of the Creative Commons Attribution License (CC BY). The use, distribution or reproduction in other forums is permitted, provided the original author(s) and the copyright owner(s) are credited and that the original publication in this journal is cited, in accordance with accepted academic practice. No use, distribution or reproduction is permitted which does not comply with these terms. 